\title{
Application of Virtual Instrument Technology in Sensor Experiment
}

\author{
Yanhua Lei $^{\mathrm{a}^{*}}$, Xinming Hou ${ }^{\mathrm{b}}$, Yan $\mathrm{Bao}^{\mathrm{c}}$ and Hongtao $\mathrm{Yu}^{\mathrm{d}}$ \\ College of Automation, Shenyang Institute of Engineering, Shenyang Liaoning110136, China \\ alyhw127@163.com, bhou_xinming@sina.com, 'dianzixinxi2004@163.com, \\ dneu970773@sohu.com \\ * The corresponding author
}

\section{Keywords: Sensor experiment; LabVIEW; Virtual experiment platform; Teaching}

\begin{abstract}
Through introducing the sensor experiment course, this paper analyzes some shortcomings of the current sensor experiment course. Combining virtual instrument technology, virtual experiment of sensor is carried out. The sensor virtual experiment platform is designed by LabVIEW and applied to the teaching of sensor experiment course. This platform promotes students' learning and mastering of sensor theoretical knowledge.
\end{abstract}

\section{Introduction}

With the development of computer technology, information technology and network technology, sensor technology and application are developing rapidly. The traditional sensor teaching, especially the practice teaching, is in urgent need of reform and innovation. The problems of traditional sensor experiment teaching are mainly reflected in two aspects: the first is the old teaching content, the verifying experiment mainly, the serious disconnection with the engineering practice, and the two is the monotonous and boring teaching method, the experiment means and the equipment fall behind. Sensor technology and application is a professional course for automatic control, mechatronics and electronics, computer and other specialties. The content of this course is scattered and applied, and practice teaching is particularly important. However, the backwardness of traditional experimental methods makes the practice teaching link only two kinds of choice: either using some special experimental equipment to do some verifying experiments, understand the working principle of some simple sensors, or buy some industrial sensors as teaching. The shortcoming of the former is that the students only contact with the special experimental equipment can only be a few simple sensors which are different from the actual industrial sensors, and most of them are verifying experiments. Through this kind of experiment, the students can only understand the principle of the sensor, and know little about the practical application. The latter, because the application of various industrial sensors is often in the industrial control process or a certain measurement and control object, the output of the sensor needs corresponding signal processing and display. Therefore, there are great shortcomings in the traditional experiments.

\section{Virtual instrument and LabVIEW}

The virtual instrument simulates the control panel of the traditional instrument by using the display function of the PC computer display, expresses the output detection result in many forms, and realizes the operation, analysis and processing of the signal data by the powerful software function of the PC computer, and completes the acquisition, measurement and adjustment of the signal by the I/O interface device. A computer system for testing functions.

At present, the most famous virtual instrument system is LabVIEW of NI. Its most widely applied field is the field of measurement and control. LabVIEW uses graphical language programming to build programs in the form of a block diagram. The device icons are basically the same as most of the icons used by scientists and engineers. This makes the programming process and the thinking process very similar. LabVIEW provides a large number of functions and subroutines to support users' tasks, as well as some special programs such as GPIB device control, VXI bus control, serial port device control, and 
data analysis, display and storage. LabVIEW also provides a toolbox dedicated to program development that allows users to set breakpoints and dynamically execute programs to observe the data transmission process for convenient debugging. At the same time, LabVIEW can call the functions of the Windows dynamic connection library and the user defined dynamic connection library to solve LabVIEW driving words in the use of the hardware supported by some companies. LabVIEW CIN node enables users to use program modules compiled by other languages, making LabVIEW a completely open development platform. In addition, LabVIEW also directly supports dynamic data exchange (DDE), structured query language (SQL), TCP and UDP network protocols.

\section{The Teaching of Sensor Experiment Course}

Traditional sensor experiment teaching has always been considered as a supplement and supplement to classroom teaching, and is subordinate to classroom teaching. The understanding of the experimental teaching of the sensor is not enough to overemphasize the guidance of the theory, ignore the activism of the practice, regard the experimental teaching as the appendage of the theory teaching, and show the theory, the heavy knowledge, the light practice, the light engineering, the teacher mainly in the experiment teaching, and the students' passively according to the teacher's demonstration method or the experimental textbook rules. The experimental results are verified by the determined steps. The teacher teaches what and how to teach. It does not consider the needs of the development of the professional training, nor does it consider the individual interests of the training objects. It makes the students in a large degree in the passive learning situation, and the students' enthusiasm, initiative and creativity are strangled, and it is difficult to realize the students. The main body status and personality differences in teaching activities, lack of comprehensive training of innovative spirit and creativity, are also not conducive to the improvement of experimental efficiency.

At present, according to the requirements and characteristics of the theoretical course, the experimental course of sensors is closely related to the professional teaching materials, and the focus of the teaching is highlighted, with the development, comprehensiveness and research in one. The experiments include: full bridge performance test of metal strain gauge, performance and application of differential transformer, experiment of vibration measurement, displacement characteristic experiment of hall type sensor, vibration experiment of piezoelectric sensor and $\mathrm{K}$ type thermocouple test. In addition to the basic experiments, a variety of derivatization experiments can be carried out. These experiments are done on the CSY2000 series sensor and detection technology experimental platform. Most of the sensors on the experimental platform are transparent, the conditioning circuit is clearly identified, including dozens of sensors and test modules, and the students can also make sensor modes according to the needs. The board provides the students with greater creative space. Through experimental training of pickup, conversion, analysis and other processes in the experiment, it helps students to strengthen the understanding of book knowledge and improve the basic operational skills and hands-on abilities as a scientific and technical worker.

\section{Design of Virtual Sensor Experimental Platform}

The design of sensor virtual experiment mainly includes the principle of the sensor, the structure of the sensor, the computer simulation design of the related experimental circuit, and the interactive design of the experiment process of the sensor and the computer network of the experiment. It is an important part of the content of the sensor experiment teaching. The virtual sensor virtual laboratory based on virtual instrument technology can make up for the shortcomings of the existing traditional sensor experiments, so that students can better understand the working rules and work characteristics of the sensor.

The virtual experiment platform of sensors is mainly matched with PC and sensor technology. The system PC uses the Windows XP operating system as the working platform, communicates with the sensor experiment platform through the USB interface, uses the virtual instrument technology to compile the control software to collect the real-time data, then uses the software to analyze and process 
the collected data dynamically or static, and then generates the coordinate curve on the PC display screen. These data are saved in file form, and the experimental records are formed in the experimental database.

The virtual instrument is used to simulate the experiment process of the sensor. This design successfully simulates the whole experiment process of multiple sensor experiments, and simulates the possible errors in the actual operation, which makes the simulated experimental data produce different degrees of error, and can be used for the experimenter to carry out the error analysis and so on. . In addition, the system can fully realize the experimental process, principles, steps, data processing, generate tables, generate curves and compare with ideal values. The data were shown to the experimenter in a simple and clear way.

This design is based on the experiment selection program. When the program starts to run into the landing interface, it can choose to use the existing account login or registered account - the experimental selection interface - the experimental procedure - the experiment principle - the experiment process - the data record - the data preservation - the retesting.

In many fields, resistance strain gauges are widely used in a variety of fields, which are popular as a strain gauge, so students should have a corresponding understanding of it as a student. This experiment is designed to enable students to understand the working principle and application of resistance strain gauges and master the strain gauge measuring circuit. As shown in Figure 1, it is a single arm bridge experiment program.

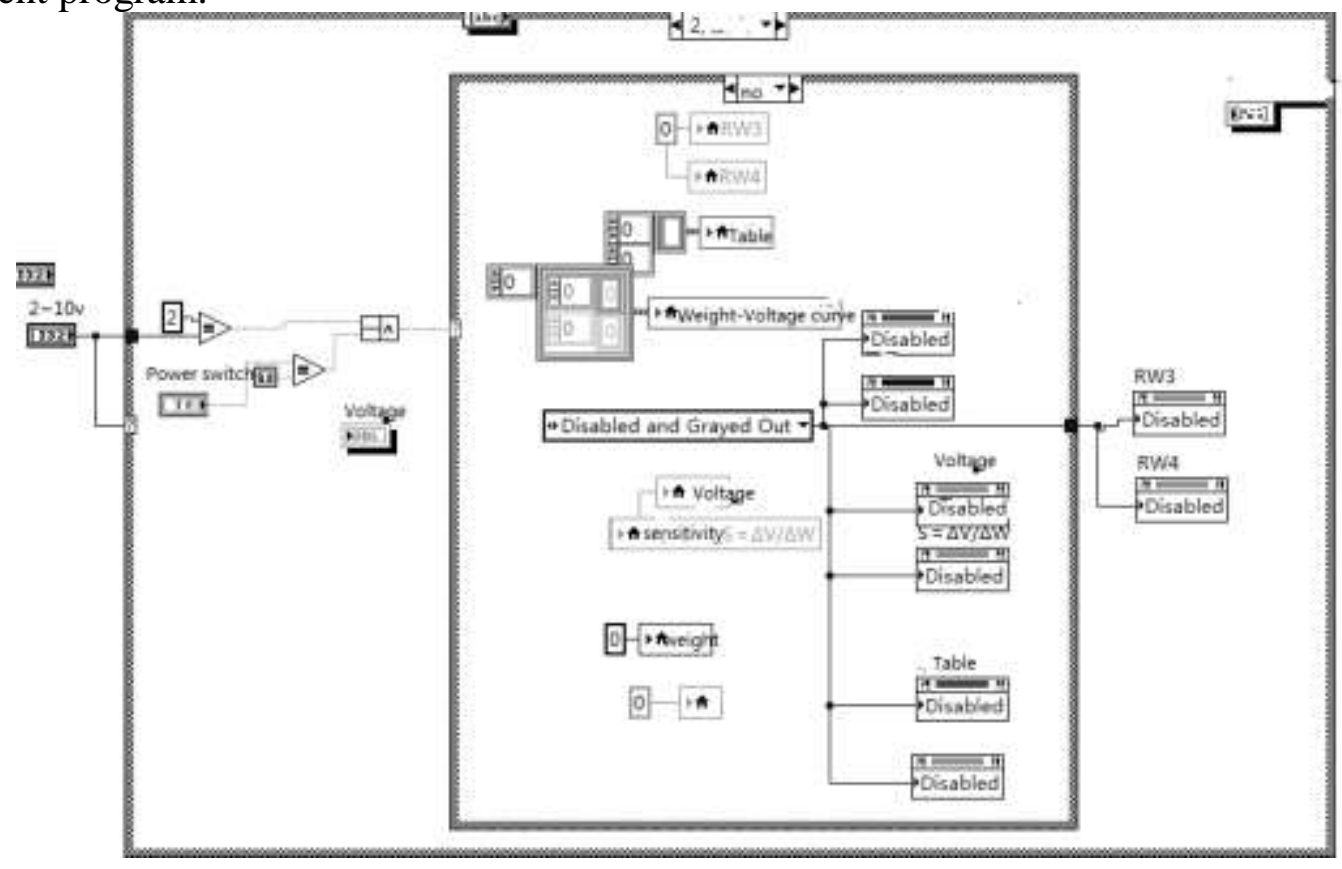

Figure 1. Finite Performance test of single arm bridge with strain gauge

When you need to save the data, the data save function, when the click data is saved, because the mechanical action to save the control record is triggered, after the mouse is loosened, it enters the event structure, starts to save, first inserts the array from the numeric value, and then calls the LabVIEW inside the generated spreadsheet Vi to pass the road. The path is split and the text formatted.Txt is saved. As shown in Fig. 2, the preservation procedure for experimental data is recorded. 


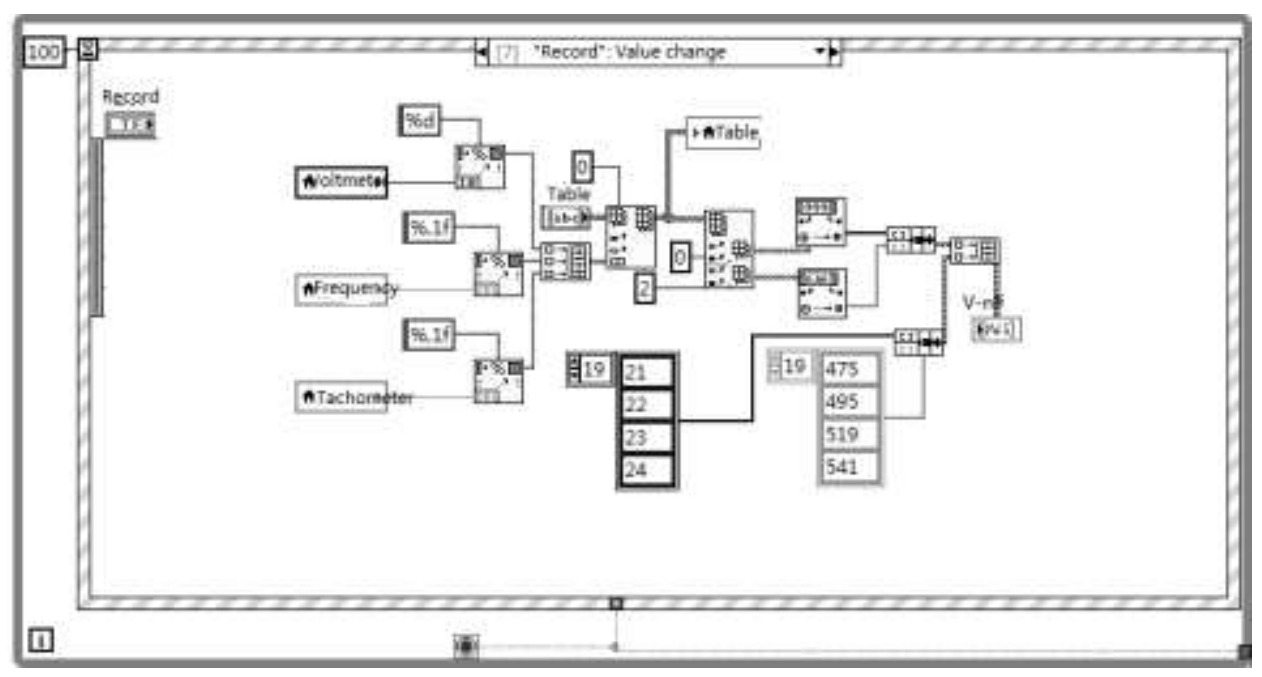

Figure 2. Finite Data recording program diagram

\section{Summary}

Instead of traditional instruments, the virtual instrument sensor experiment technology platform is used to carry out experiments in teaching and reduce the expenditure and loss of experimental equipment. This design focuses on the authenticity of the experimental process. The simulation data processing and experimental process simulation are mainly written to make the user feel the same experience as the traditional instrument, and it is superior to the experimental results and data processing analysis of the traditional instruments.

\section{References}

[1] Y.J Lv: Practical course of sensor technology (Machinery Industry Press, China 2012). (In Chinese)

[2] Z.A. Jaffery and A.K. Dubey: The role of educational software as a support for teaching and learning conversations, Computers \& Education, 2003, Vol.43 (1), pp.179-191

[3] Mohammad Abu Zalata, Tariq M. Younes, Mohammad A. K. Alia.: Development of Equivalent Virtual Instruments to PLC Functions and Networks. Journal of Software Engineering and Applications . 2011 (03)

[4] Essick J: Hands-on introduction to LabVIEW for scientists and engineers. 2012

[5] Berkay Erder,Aynur Akar: Remote accessible laboratory for error controlled coding techniques with the labview software. Procedia - Social and Behavioral Sciences . 2010 (2)

[6] Peng L. Cost heterogeneity and peak prediction in collective actions [J]. Expert Systems With Applications, 2017, 79: 130-139. 